\title{
Analysis of the characteristics and trusted security of cloud computing
}

\author{
Sanjay Ram M Vijayaraj A \\ Associate Professor, Department of MCA, Adhiyamaan College of Engineering, \\ Hosur,Tamilnadu,India-635 109. \\ sanjayramm@gmail.com \\ Associate Professor, Department of IT Saveetha Engineering College, \\ Thandalam,Chennai-602 105. \\ satturvijay@yahoo.com
}

\begin{abstract}
Cloud computing allows people the way to sharing distributed resources and services that belong to various organizations and sites. The cloud computing has its own concept, technical, economic and user experience characteristics. The service oriented, loose coupling, strong fault tolerant, business model and ease use are main characteristics of cloud computing. In this paper, proposed a method to build a trusted computing environment for cloud computing system by integrating the trusted computing platform into cloud computing system and pay attention to the security requirements in cloud computing environment. Some important security services, including authentication, confidentiality and integrity, are provided in cloud computing system.
\end{abstract}

\section{Keywords}

cloud computing; grid computing; service oriented; loose coupling; strong fault tolerant; business pattern; ease use, trusted computing platform; trusted computing; trusted service

\section{INTRODUCTION}

Cloud computing is TCP/IP based high development and integrations of computer technologies such as fast micro processor, huge memory, high-speed network and reliable system architecture. Without the standard inter-connect protocols and mature of assembling data center technologies, cloud computing would not become reality too.Cloud computing is a term used to describe both a platform and type of application. A cloud computing platform dynamically provisions, configures, reconfigures, and de-provisions servers as needed. Servers in the cloud can be physical machines or virtual machines. Advanced clouds typically include other computing resources such as storage area networks (SANs), network equipment, firewall and other security devices. Cloud computing also describes applications that are extended to be accessible through the Internet. These cloud applications use large data centers and powerful servers that host Web applications and Web services. Anyone with a suitable Internet connection and a standard browser can access a cloud application. A cloud is a pool of virtualized computer resources. A

DOI : $10.5121 /$ ijccsa.2011.1305 
cloud can host a variety of different workloads, including batch-style back-end jobs and interactive, user-facing applications. Allow workloads to be deployed and scaled-out quickly through the rapid provisioning of virtual machines or physical machines. It is Support redundant, self-recovering, highly scalable programming models that allow workloads to recover from many unavoidable hardware / software failures. Monitor resource use in real time to enable rebalancing of allocations when needed. Cloud computing environments support grid computing by quickly providing physical and virtual servers on which the grid applications can run. Cloud computing should not be confused with grid computing. Grid computing involves dividing a large task into many smaller tasks that run in parallel on separate servers. Grids require many computers, typically in the thousands, and commonly use servers, desktops, and laptops. Clouds also support non-grid environments, such as a three-tier Web architecture running standard or Web 2.0 applications. A cloud is more than a collection of computer resources because a cloud provides a

\begin{tabular}{|l|l|l|}
\hline \multicolumn{1}{|c|}{ Characteristics } & $\begin{array}{l}\text { Cloud } \\
\text { Computin } \\
\text { g }\end{array}$ & $\begin{array}{l}\text { Grid } \\
\text { Computing }\end{array}$ \\
\hline Service Oriented & Yes & Yes \\
\hline Strong Fault Tolerant & Yes & Half \\
\hline TCP/IP Based & Yes & Half \\
\hline High Security & Half & Half \\
\hline Loose Coupling & Yes & Half \\
\hline Virtualization & Yes & Half \\
\hline Ease Use & Yes & Half \\
\hline Commercial Pattern & Yes & No \\
\hline
\end{tabular}

mechanism to manage those resources. Management includes provisioning, change requests, reimaging, workload rebalancing, de-provisioning, and monitoring. Beside the web email, the Amazon Elastic Compute Cloud (EC2) [2], Google App Engine [3] and Salesforce's CRM [4] largely represent a promising conceptual foundation of cloud services. The services of cloud computing are

TABLE 2.1 Cloud Computing Vs. Grid Computing Characteristics

Service (PaaS), and Software-as-a-Service (SaaS) [5, 6]. Cloud computing also is divided into five layers including clients, applications, platform, infrastructure and servers. The five layers look like more reasonable and clearer than the three categories [7].

This paper tries to summarize general characteristics and trusted security of cloud computing which will help the development and adoption of this rapidly evolving technology. There will be full war on cloud computing among big giants including Google, Microsoft, Apple, Amazon, IBM, Oracle and HP etc. It is hard to predict who will win the war. It is also hard to predict the impact of cloud computing on both information technology and society. It gives a more comprehensive analysis of all the characteristics of Cloud Computing. The clouds are a large pool of virtualized resources which are ease to use and access. There are ten characteristics of cloud 
computing in their sum-up: user friendliness, virtualization, Internet centric, variety of resources, automatic adaptation, scalability, resource optimization, pay-per-use, service SLAs (ServiceLevel Agreements) and infrastructure SLAs.

The characteristics of cloud computing are much more complex in [11]. Cluster's resources are located in single administrative domain with single entity. Resources of grid system are distributed and located in administrative domain with multi entity and management policies. And cloud computing platform possesses characteristics of both cluster and grid. The cloud computing platform provides services to users without knowing much about the infrastructure.

\section{II.CHARACTERISTICS OF CLOUD COMPUTING}

The comparable characteristics of cloud computing and grid computing are listed in Table I. The "yes" and "no" stand for cloud computing or grid computing has the special characteristic or not. The "half" means not owning the whole characteristic to a certain extent. This paper doesn't pay much attention on the similarities and difference between them and focuses on the essential characteristics of cloud computing [6].

The cloud computing, grid computing, High performance computing (HPC) or supercomputing and data center computing all belong to parallel computing. HPC focuses on scientific computing which is computing intensive and delay sensitive. So high processing performance and low delay are the most important criteria in HPC. Grid computing is based on HPC center. Many connected HPC centers form a large grid which owns a powerful underlying concept - service oriented architectures (SOA). Some other creative and impressive concepts like utility computing and autonomic computing do not come into reality. The cloud computing which is based on data center is much more widely accepted than grid computing. Data center which doesn't only pursue powerful processing performance and low delay is more balanced than HPC center.

\subsection{Service oriented}

The service oriented concept is similar to but more practical than the concept of SOA in grid computing. Abstraction and accessibility are two keys to achieve the service oriented conception. Through virtualization and other technologies, the underlying architecture is abstracted without exposing much to user. So it is opacity to cloud user. Abstraction reduces both the need for cloud user to learn the detail of cloud architecture and the threshold of application development. At the same time, the key elements of underlying architecture can be simply accessed by cloud user. Cloud user can consume all the capacity easily by exploring system parameters such as processing performance and storage capacity. In general, according to the type of provided capability, the services of cloud computing are broadly divided into three categories: Infrastructure-as-a-Service (IaaS), Platform-as-a-Service (PaaS), and Software-as-a-Service (SaaS) $[5,6]$. Infrastructure-as-a-Service is the delivery of huge computing resources such as the capacity of processing, storage and network. Taking storage as an example, when a user use the storage service of cloud computing, he just pay the consuming part without buying any disks or even knowing nothing about the location of the data he deals with. Sometimes the IaaS is also called Hardware-as-a-Service (HaaS). Platform-as-a-Service generally abstracts the infrastructures and supports a set of application program interface to cloud applications. It is the middle bridge between hardware and application. Because of the importance of platform, many 
big companies want to grasp the chance of predominating the platform of cloud computing as Microsoft does in personal computer time. The well known examples are Google App Engine [3] and Microsoft's Azure Services Platform [15]. Software-as-a-Service aims at replacing the applications running on PC. There is no need to install and run the special software on your computer if you use the SaaS. Instead of buying the software at a relative higher price, you just follow the pay-per-use pattern which can reduce you total cost. The concept of SaaS is attractive and some software runs well as cloud computing, but the delay of network is fatal to real time or half real time applications such as 3D online game.

Apple's App store [20] is a creative and famous cloud computing in wireless area. Software services are sold in pay-per-use style. But running on terminals such as $3 \mathrm{G}$ phones instead of huge data centers is different from SaaS. The electric books resources in Amazon [21] are also services in cloud computing. These services hardly have substitution and building another EC2 is much easier than owning so many electric resources. In online game area, powerful game servers supply the interactions of millions game players. Game players use the capability of cloud computing without much waking up to this technology.

\subsection{Loose coupling}

The loose coupling is the technical fundamental of cloud computing and goes beyond the loose coupling method of application interaction. Through virtualization or other technologies, the infrastructures are separated in logic or physic. The behavior of one part hardly affects other parts. For example, the platform is an abstract layer which can isolate different applications running on it. Most important of all, whole cloud computing runs in a client-server model. The clients or cloud users connect loosely with servers or cloud providers. All the users have almost no data or control dependence. The independent cloud users can induce many other features such as stateless, scalability and strong fault tolerant. Software services are sold in pay-per-use style. But running on terminals such as $3 \mathrm{G}$ phones instead of huge data centers is different from SaaS. All these services are as important an opposite example is the tight coupling of HPC systems which focus on solving scientific problems. Usually, there are too many data dependences or global synchronizations in different iterations to bear the high delay among computing nodes. This kind of systems use high speed network e.g. InfiniBand instead of industrial standard Ethernet which is much cheaper and widely supported. It is hard to imagine spending tens of minutes to achieve a global synchronization in HPC. But in cloud computing one time of global data synchronization may cost several hours or even several days.

\subsection{Strong fault tolerant}

There are many fault tolerant methods in parallel computing. At low-level, there always existsome fault correction mechanisms with specific hardware. At high-level, many specific applications are studied with methods aiming at algorithms. Checking point is one of the most effective methods at middle-level. In large scale parallel computer systems, the interval of two failures may be shorter than application execution time. For example, some scientific computing applications run for weeks or even longer but there may be several trivial or fatal errors during the whole runtime.

The fault tolerant technology becomes critical in this condition. Otherwise it has only less chance to complete the time consuming computing tasks. Because a minimum error is unacceptable and 
redoing costs too much time in many scientific applications, so the whole computation states which are saved periodically on stable storage will roll back to a special checking point if an error occurs. It is unnecessary to keep the whole states of cloud computing systems. There is almost no dependence between two transactions. The failure of one transaction does not affect the other one and partly failure of system will not cause chain reaction. There are mainly four places where faults maybe occur in cloud computing: provider-inner, provider-across, provider-user and useracross.

If a fault occurs in provider, the backup or redundancy of provider will substitute for the failed part. Stop services and restart are another common method if the services are not on time or urgent. If a fault occurs among providers, the provider-across transaction will be canceled and return with an error hint. Redirecting to other providers is a universal method which involves load balance of whole cloud system. Fortunately, there are only fewer transactions, which are caused by background management in the main, involving more than one provider. It needs only to run background management one time per day or even per week.

\subsection{Ease Use}

Ease use is not only simple but also elegant. User experience which belongs to the subject of human computer interaction is an important criterion when evaluating whether an application is successful or not. In cloud computing, user experience improves a lot than its ancestors like grid computing. The cloud service is a means toward the end of providing a good experience for cloud user. The valuable services should be easily accessed by cloud user. The core of user experience is achieving ease use. There are three reasons why cloud computing should be ease use: First, most cloud providers offer Internet-based interfaces which are simpler than other application program interfaces (API). These interfaces are simple and elegant enough to hide the business processing behind. The interfaces can stay the same ignoring whether the business processing has changed or not.

Second, user experience of web applications is full studied. So the user interfaces are independent of content. The development of web application has a full suite of flow which can be divided into three stages including user need analysis, function design and program implementation. In top-down method, the user experience design is the fundamental of whole function design. The facets of the user experience are useful, usable valuable, desirable, findable, credible and accessible [23].

Third, the web 2.0 increases the interactions between web users and providers. The web was originally designed to transport hypertext. As the rapid and rich developments of increasingly sophisticated contents are appearing, web is usually used as a remote software interface. The web 2.0 is supposed to be the continuum of user experience and blurs the line between software and the Internet. The emerging AJAX technology makes web applications and services are becoming more software-like [24]. All these reduce information technology overhead for the end user. Search in the Internet is very simple: a web page with an input text and a confirm button.

\subsection{Other characteristics}

The other important characteristics are TCP/IP based, virtualization and high security. TCP/IP gives reliable delivery, a connection-oriented service between remote applications. TCP/IP is widely used in cloud computing. Although the network protocols may be private in the back end 
of data center, most cloud users connect through TCP/IP. The HTTP protocol over TCP/IP or Internet inspires the user experience characteristics. Cloud resources are often virtualized as a service over the Internet. Up to the present, many cloud computing infrastructures consist of data centers. Data center uses virtualization technologies which abstract the commonness of infrastructure in different levels. High security of cloud computing is achieved mainly through three ways. First, the loose coupling makes cloud computing system run well when part of it is destroyed. Second, the abstraction, virtualization and privation of cloud provider avoid exposing the details of corresponding implementations. Third, technology cooperating with law is the guard of cloud computing.

\section{TRUSTED SECURITY IN CLOUD COMPUTING SYSTEM USING TCP}

The trusted computing mechanism can provide a way that can help to establish a security environment. The model of trusted computing is originally designed to provide the privacy and trust in the personal platform and the trusted computing platform is the base of the trusted computing. Since the internet computing or network computing has been the main computing from the end of the last century, the model of trusted computing is being developed to the network computing, especially the distributed systems environment. The cloud computing is a promising distributed system model and will act as an important role in the e-business or research environments. Web service technology have developed as quickly and have been used broadly, cloud computing system could evolve to cloud computing service, which integrates the cloud computing with web service technology. So we could extend the trusted computing mechanism to cloud computing service systems by integrating the TCP into cloud computing system. The network computing environment, trust will go on to envision a connected, digital world in which trusted entities would interact with one another in much the same way individuals and businesses interact in traditional commercial relationships. "The digital universe requires that parties to a common transaction be able to trust that their mutually agreed upon intents will be fulfilled and their rights protected. For true commerce automation to exist, trading partners must know what to expect from each other's systems."[26]. Trusted computing, therefore, must provide the basis for trusted transactions to occur, and trusted computing technologies must allow stakeholders to express policies and have those policies negotiated and enforced in any execution environment.

\subsection{Authentication cloud computing environment with TCP}

The cloud computing environment, different entities can appeal to join the CLOUD. First step to prove their identities to the cloud computing system administration. Because cloud computing should involve a large amount of entities, such as users and resources from different sources, the authentication is important and complicated. Considering these, we use the TCP to aid to process the authentication in cloud computing. The TCP is based on the TPM. The TPM is a logic independent hardware. It can resist the attack from software, and even the hardware attack. The TPM contain a private master key which can provide protect for other information store in cloud computing system. Because the hardware certificate can store in TPM, it is hard to attack it. So TPM can provide the trust root for users. Since the users have full information about their identity, the cloud computing system can use some mechanism to trace the users and get their origin. Because in the TCP the user's identity is proved by user's personal key and this mechanism is integrated in the hardware, such as the BIOS and TPM, so it is very hard to the user 
to make deceiving for their identity information. Each site in the cloud computing system will record the visitor's information. So by using the TCP mechanism in cloud computing, the trace of participants can be known by the cloud computing trace mechanism.

\subsection{Data Security in cloud based on TCP}

With the TCP, the different entities can communicate in a security way. The TCP generate random numbers and then create session keys. The random keys created by physical hardware have the security characteristics better than those generated just by software programs. The security communication protocols use the system in cloud to call TSS to use the TPM. Then TPM provides the encryption key and session key to the communicators in cloud computing. With its computing capacity, TPM can burden few computation works from CPU and improve the performance. The important data stored in the computer can be encrypted with keys generated by the TPM. When accessing to these data, the users or applications should pass firstly the authentication with TPM, and encryption keys are stored in the TPM, which makes it hard to attack these keys. To prevent the attack for integrity of data, the hash function in TPM is used. The TPM will check the critical data in a certain interval to protect the integrity of data. The processes of encryption and integrity check use TSS to call the function of TPM.

\subsection{The Trace of the User's Behavior}

Since the users have full information about their identity, the cloud computing system can use some mechanism to trace the users and get their origin. Because in the TCP the user's identity is proved by user's personal key and this mechanism is integrated in the hardware, such as the BIOS and TPM, so it is very hard to the user to make deceiving for their identity information. Before the distributed machine cooperates to do something, they should attest their local information to the remote site. When the user login the cloud computing system, his identity information should be recorded and verified at first. Each site in the cloud computing system will record the visitor's information. So if the TCP mechanism is integrated into the cloud computing, the trace of the participants, including the users and other resources, can be knew by the cloud computing trace mechanism. Then if the participants do some malicious behavior, they will be tracked and be punished. In order to achieve the trusted computing in the cloud computing system, we should have the mechanism to know not only what the participants can do, but also what the participant have done. So the monitoring function should be integrated into the cloud computing system to supervise the participants' behavior. In fact, reference monitors have been used in the operation system for more than several decades, and it will be useful in cloud computing too.

\section{CONCLUSION AND FUTURE WORK}

The Understanding a technical area as complex as cloud computing is not easy and requires identifying its fundamental characteristics. Clear concepts and terminology into cloud computing help but do not entirely solve the problem of how to design, develop and adopt a cloud computing system. This paper discusses the characteristics and trusted security in of cloud computing. The conception service oriented characteristic abstracts the details of cloud computing implementation. The loose coupling and strong fault tolerant stand for the main technical characteristics. Owning the business model is the key differentiation compared with 
other academic researches and helps cloud computing flourishing. The ease use user experience characteristic helps cloud computing being widely accepted by non computer experts. We believe that these characteristics expose the essential of cloud computing and the development and adoption of this evolving technology will benefit from our work. The analyzed trusted computing in the cloud computing environment and the function of trusted computing platform in cloud computing. The advantages of proposed approach are to extend the trusted computing technology into the cloud computing environment to achieve the trusted computing requirements for the cloud computing and then fulfill the trusted cloud computing. TCP is used as the hardware base for the cloud computing system. In this design, TCP provides cloud computing system some important security functions, such authentication, communication security and data protection. The TCP provides cloud computing a secure base for achieve trusted computing. As a part of the future work to get detail of each characteristics and trusted security services especially the inner technologies and the evaluation of cloud services is an interesting and valuable research subject. It will make the actual design more practical and operational in the future.

\section{REFERENCES}

[1] IBM, "Google and IBM Announced University Initiative to Address Internet-Scale Computing Challenges,"http://www-3.ibm.com/press/us/en/pressrelease/22414.wss.

[4] Salesforce, "CRM”, http://www.salesforce.com/.

[5] searchcloudcomputing.com,"What is cloud computing?" http://searchcloudcomputing.techtarget.com/sDefinition/0,sid201_gci1287881,00.html.

[6] L.M. Vaquero, L.R. Merino, J. Caceres, and M. Lindner, "A break in the clouds: towards a cloud definition,” ACM SIGCOMM Computer Communication Review, v.39 n.1, 2009.

[7] Wikipedia, "Cloud computing," http://en.wikipedia.org/wiki/Cloud_computing.

[8] J. Geelan, "Twenty one experts define cloud computing. Virtualization," Electronic Magazine, http://virtualization.sys-con.com/node/612375.

[9] D. Farber, "Oracle's Ellison nails cloud computing," http://news.cnet.com/ 8301-13953_310052188-80.html.

[10] R. Buyya, C.S. Yeo, S. Venugopal, J. Broberg, and I. Brandic, "Cloud computing and emerging IT platforms: Vision, hype, and reality for delivering computing as the 5th utility," Future Generation Computer Systems, 25 (6), pp. 599-616, 2009.

[11] D. Malcolm, "The five defining characteristics of cloud computing," http://news.zdnet.com/21009595_22-287001.html.

[12] P. Sharma, "What kinda apps are best suited for 'Cloud deployment' : 4 Solutions," http://www.techpluto.com/cloud- omputingcharacteristics/. 
International Journal on Cloud Computing: Services and Architecture(IJCCSA),Vol.1, No.3, November 2011

[13] D. Amrhein, "Forget Defining Cloud Computing," http://ibm.ulitzer.com/ node/1018801.

[14] Microsoft,“WindowsAzure," http://www.microsoft.com/windowsazure/ windowsazure/.

[15] P. Watson, P. Lord, F. Gibson, P. Periorellis, and G. Pitsilis, "Cloud computing for e-science with carmen,” IBERGRID, 2008, pp.1-5,

[16] B. Rochwerger, D. Breitgand, E. Levy, A. Galis, K. Nagin, L. Llorente, R. Montero, Y. Wolfsthal, E. Elmroth, J. C'aceres, M. Ben-Yehuda, W. Emmerich, and F. Gal'an , "The RESERVOIR Model and Architecture for Open

\section{Authors Profile:}

A.Vijayaraj is an Associate Professor in Department of Information Technology at Saveetha Engineering College. He received his Master of Computer Application in Bharathidhasan University, in 1997 and his Master of Engineering in Computer Science and Engineering from Sathyabama University at 2005. He has 12 years of teaching experience from various Engineering Colleges during tenure he was Awarded Best Teacher Award twice..He is a Member of, CSI and ISTE. He has Published 2 papers in International journal and 10 Papers in International and National Level conferences. His area of interest includes Operating Systems, Data Structures, Networks and Communication.

Sanjay Ram M : Sanjay Ram M is an Associate Professor in Department of Computer Application at Adhiyamaan College of Engineering. He received his Master of Computer Application in Annamalai University in 2000. He has 11 years of teaching experience from various Engineering Colleges. He is a Member of CSI. He has Published 5 papers in National Level conferences. His area of interest includes Operating Systems
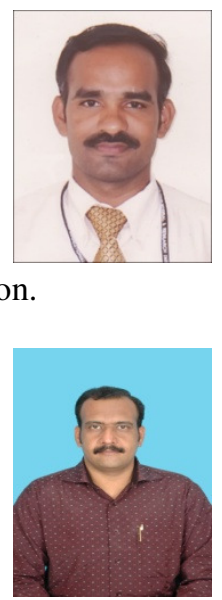